\title{
Assessing the Impact of Probiotic Supplementation and Caloric Periodization on Ultra-endurance Performance and Gastrointestinal Symptoms.
}

Justin D. Roberts ${ }^{1}$, Craig A. Suckling ${ }^{1}$, Georgia Y. Peedle ${ }^{1}$, Dan A. Gordon ${ }^{1}$, Hannah Marshall ${ }^{2}$, Lee Taylor $^{3}$ and Michael G. Roberts ${ }^{4}$. Anglia Ruskin University, United Kingdom ${ }^{1}$; University of Bedfordshire, United Kingdom²; ASPETAR, Qatar Orthopaedic and Sports Medicine Hospital, Qatar; University of Hertfordshire, United Kingdom ${ }^{4}$.

Beneficial use of probiotic (PRO) interventions on gastrointestinal endotoxemia (GE) prior to an ultra-endurance triathlon has been previously demonstrated. The prevalence of GE (and whether PRO strategies minimise gastrointestinal (GI) symptoms) relating to multi-day ultra-events is less known. Understanding if nutritional periodization strategies confer similar GI benefits also warrants investigation.

PURPOSE: To assess the impact of probiotic supplementation and caloric periodization prior to an extreme ultra-marathon on $\mathrm{Gl}$ symptoms and race performance.

METHODS: Thirty-eight healthy participants were recruited from entrants of the 2015 Marathon Des Sables (age: 42 $\pm 9 y \mathrm{rs}$; weight: $77.71 \pm 10.31 \mathrm{~kg}$; $\mathrm{VO}_{2 \max }: 52.58 \pm 8.66$ $\left.\mathrm{mL} \cdot \mathrm{kg} \cdot \mathrm{min}^{-1}\right)$, and randomly assigned to either: PRO $\left(100 \mathrm{mg} \cdot \mathrm{d}^{-1}\right.$ capsulated Lactobacillus acidophilus); CP (caloric periodization of 500kcal above habitual intake on alternate days) or control (CON) for 12 weeks pre-race. Plasma lipopolysaccharides (LPS) via Limulus Amebocyte Lysate chromogenic endotoxin quantification were determined at baseline, pre and post-race. Participants graded duration and severity of Gl symptoms through daily questionnaires. Performance times were obtained from accumulated race tracking. Data presented as mean $\pm S E$.

RESULTS: Race times (hrs:mins) were $41: 28 \pm 2: 31,45: 12 \pm 2: 05$ and $50: 43 \pm 4: 38$ for PRO, CP and CON respectively ( $p>0.05)$. Overall LPS significantly increased from baseline $\left(10.08 \pm 0.53 \mathrm{pg} \cdot \mathrm{ml}^{-1}\right)$ to pre-race $\left(13.12 \pm 0.74 \mathrm{pg} \cdot \mathrm{ml}^{-1} ; \mathrm{p}=0.001\right)$. Delta LPS prerace was not different between groups (PRO: $2.94 \pm 1.11 \mathrm{pg} . \mathrm{ml}^{-1}$; CP: $3.71 \pm 1.28 \mathrm{pg} \cdot \mathrm{ml}^{-}$ 1; CON: 2.32 $\pm 1.26 \mathrm{pg} \cdot \mathrm{ml}^{-1}$; $\left.\mathrm{p}>0.05\right)$. Similarly, delta LPS post-race was not different, despite greater reductions in both intervention groups (PRO: $-4.57 \pm 1.93 \mathrm{pg} \cdot \mathrm{ml}^{-1}$; CP: $\left.6.95 \pm 1.84 \mathrm{pg} \cdot \mathrm{ml}^{-1} ; \mathrm{CON}:-2.16 \pm 2.21 \mathrm{pg} \cdot \mathrm{ml}^{-1} ; \mathrm{p}>0.05\right)$. Gl symptom count favoured PRO 
(21.8\%) compared with CP (41.6\%) and CON (36.6\%) respectively $(p=0.001)$, although no differences for $\mathrm{Gl}$ symptom index were reported between groups $(p>0.05)$.

CONCLUSIONS: Moderate GE was evident in a UK cohort undertaking a multi-day ultra-marathon. PRO use did not significantly impact on GE prevalence, despite evidence of reduced GI symptoms. Caloric periodization appeared to favour GE recovery post-race, but was not deemed significant. 